\title{
Impact de l'emballage et de la durée de conservation sur la qualité nutritionnelle et microbiologique des biscuits de sorgho enrichis au moringa et à la spiruline
}

\author{
Laurencia T. Songré-Ouattara1, Mahamadé Goubgou ${ }^{1,2}$, Aly savadogo² \\ ${ }^{1}$ Institut de Recherche en Sciences Appliquées et Technologies (IRSAT), Département Technologie Alimentaire \\ (DTA), 03 BP 7047 Ouagadougou 03 Burkina Faso, \\ 2Université Ouaga I Pr Joseph KI-ZERBO, Unité de formation et Recherche en Sciences de la Vie et de la Terre \\ (UFR/SVT), Laboratoire LABIA 03 BP 7021 Ouagadougou 03, Burkina Faso. \\ Auteur correspondant : Email : laurenciaouattara@yahoo.fr, Tél : 0022670278633
}

Original submitted in on $6^{\text {th }}$ October 2016. Published online at www.m.elewa.orgon $31^{\text {st }}$ January 2017 http://dx.doi.org/10.4314/jab.v109i1.1

\section{RESUME}

Objectif : L'évolution de la qualité de deux types de biscuits de sorgho enrichis à $10 \%$ de moringa et à $4 \%$ de spiruline a été étudié selon deux types d'emballage, notamment l'aluminium et le plastique pendant 12 mois et comparé à un biscuit témoin afin de déterminer leur durée de vie.

Méthodologie et résultats : Plusieurs paramètres de qualité nutritionnelle (humidité, lipides, protéines, glucides, cendres, fer, zinc) et de rancidité oxydative (acidité grasse, indice de peroxyde) et microbiologique (coliformes totaux, coliformes fécaux et flore aérobie mésophile totale) ont été analysés durant la conservation à température ambiante utilisant des méthodes standards. Les résultats montrent qu'il n'y a pas de changement significatif pour les paramètres nutritionnels dans chaque type de biscuit pour les deux types d'emballage étudiés durant la conservation. Pour les types de microorganismes étudiés, seule la flore aérobie mésophile totale présente des concentrations différentes et très variables dans le temps et comprises entre 2,1 log (UFC/g) à 5,1 log (UFC/g) attestant ainsi une contamination des emballages utilisés. Les taux d'acidité grasse et d'indice de peroxyde dans les biscuits sont variables et particulièrement plus élevés pour les biscuits les plus riches en lipides comme le sorgho enrichis au moringa. Toutefois, les indices de peroxyde initiaux très élevés dans les différents types de biscuits laissent supposer un début d'oxydation qui évolue peu durant la conservation en dépit de l'emballage utilisé.

Conclusion et application des résultats : Cette étude a permis de déterminer que les biscuits de sorgho enrichis pouvaient être conservés dans les emballages de type aluminium et de type plastique pendant 12 mois. Ces résultats fournissent ainsi des informations techniques aux acteurs du secteur de l'agroalimentaire.

Mots clés : biscuit, sorgho, enrichissement, qualité 
Songré-Ouattara et al ., J. Appl. Biosci. 2017 Impact de l'emballage et de la durée de conservation sur la qualité nutritionnelle et microbiologique des biscuits de sorgho enrichis au moringa et à la spiruline

Impact of the packaging and conservation on the quality, nutritional and microbiological of sorghum cookies fortified with moringa and Spirulina

Objective: Changes in the quality of two types of sorghum biscuits enriched with $10 \%$ moringa and $4 \%$ spirulina were studied in two types of packaging, namely aluminum and plastic for 12 months and compared to $A$ control biscuit to determine their lifetime.

Methodology and results: several parameters of nutritional quality (moisture, fat, protein, carbohydrate, ash, iron, zinc) and oxidative rancidity (FAT acidity, peroxide index.) and microbiological (total coliforms, fecal coliforms and total flora aerobic mesophilic) were analyzed during the conservation period at room temperature using standard methods. The results show that there was no significant change for the nutritional parameters in each type of cookie for the two types of packaging. For the types of microorganisms studied, only the total flora aerobic mesophilic present in different concentrations and very variable in time and between $2.1 \mathrm{log}(\mathrm{cfu} / \mathrm{g})$ to $5.1 \mathrm{log}(\mathrm{cfu} / \mathrm{g})$ indicating a contamination of packaging used. Rates of fat acidity and peroxide index in the cookies were variable and especially higher for cookies that are richest in lipids as sorghum fortified with moringa. However, initial very high peroxide indexes in different types of cookies suggests a beginning of oxidation that has changed little during the conservation despite the packaging used.

Conclusion and application of the findings: this study determined that fortified sorghum cookies could be kept in the aluminum or plastic type of packaging for 12 months. These results thus provide technical information to stakeholders in the food industry sector.

Keywords : biscuit, sorghum, enrichment, quality

\section{INTRODUCTION}

La consommation des biscuits est devenue de nos jours très populaires à travers le monde (Agu et Okoli, 2014). Les biscuits sont des produits céréaliers secs, essentiellement composés de farine de féculent et de quantité appréciable de matière grasse et de sucre (O'Brien et al., 2003). II existe de nombreuses recettes de biscuits qui sont basées sur la variabilité des proportions des ingrédients majoritairement utilisés et l'inclusion de nouveaux ingrédients, permettant ainsi de les catégoriser en 10 groupes (Manley, 2000). Traditionnellement, les biscuits et le pain sont élaborés à partir des farines de blé, une céréale cultivée dans plusieurs parties du monde mais qui est importée par les pays dont le climat n'est pas favorable à la culture de cette céréale (Okpala et al., 2013). Pour ces derniers, la dépendance à l'utilisation des farines de blé est une contrainte majeure dans la production des biscuits. Par conséquent, l'utilisation des farines de céréales locales comme substitut à la farine de blé présente un grand intérêt socioéconomique pour ces pays. En effet, le potentiel d'utilisation des farines de céréales sans gluten et ou des légumineuses comme substitut total ou partiel à la farine de blé dans la production des biscuits a été rapporté par plusieurs auteurs
(Agu et al., 2007, Ratika et al., 2012 ; Okpala et al., 2013 ; Agu and Okoli., 2014 ; Songré-Ouattara et al., 2016). De plus, la modification des formules par substitution des farines de céréales par des ingrédients nutritionnellement intéressants est une alternative permettant d'améliorer la valeur nutritionnelle des biscuits (Handa et al., 2012; Yadav et al., 2012; Chakraborty et al., 2011; Gajera et al., 2010) avec des modifications organoleptiques désirables. Dans les stratégies de lutte contre la malnutrition, la fortification, l'enrichissement ou la complémentation des produits de grande consommation occupent une place centrale. A cet effet, le développement et l'utilisation des biscuits enrichis, notamment riches en protéines, en lipides et en certains micronutriments essentiels comme le fer, le zinc ou la vitamine A pourrait contribuer à réduire les risques de malnutrition protéinoénergique ou de carences nutritionnelles et de ce fait à améliorer le statut nutritionnel et la santé des enfants en particulier dans les pays en développement. Récemment, les biscuits sont considérés comme des aliments fonctionnels, c'està-dire des aliments qui renferment des éléments bénéfiques pour la santé. Ainsi, ces derniers peuvent 
fournir journalièrement des substances nutritives et protectrices pour le maintien ou l'amélioration de la santé. Au Burkina Faso, la malnutrition de type pluricarentiel et les carences spécifiques (fer, vitamine A, zinc etc.) sont des questions majeures de santé publique qui touchent les populations les plus à risque, notamment les enfants, les femmes enceintes et allaitantes. II ressort des enquêtes démographiques et de santé que l'émaciation, le retard de croissance, l'insuffisance pondérale et l'anémie nutritionnelle touchent respectivement $16 \%$, $35 \%, 26 \%$ et $88 \%$ des enfants de moins de 5 ans (INSD, 2012). C'est ainsi que de nombreux efforts sont réalisés pour améliorer l'accessibilité d'aliments de complément nutritionnellement adéquat pour les jeunes enfants. Afin de contribuer à la lutte contre ce

\section{MATERIEL ET METHODES}

Site de l'étude : Les productions de biscuits de sorgho ont été réalisées à l'atelier de production du Département Technologie Alimentaire (DTA) de l'Institut de Recherche en Sciences Appliquées et Technologies (IRSAT) à Ouagadougou, au Burkina Faso.

Matières premières et ingrédients utilisés : Les grains et sorgho de la variété Gampèla étaient achetés auprès d'un producteur. Ces grains étaient transformés en farine après nettoyage, décorticage, vannage, lavage et épierrage, séchage, mouture et tamisage utilisant un tamis de maille inférieur à $200 \mu \mathrm{m}$. Les ingrédients comme la matière grasse, le sucre, le lait en poudre, les œufs, le sel, le sucre vanillé étaient achetés dans une alimentation de la ville de Ouagadougou. La poudre de feuilles séchées de moringa était obtenue auprès de l'association des producteurs de la Sissili pour l'Ecogestion des ressources naturelles (APSERN) et la poudre fléau, deux formulations de biscuits de sorgho enrichies avec la poudre de feuille séchée de moringa (Moringa oleifera) et avec la poudre de spiruline (Spirulina platensis) une micro-algue localement produite, respectivement à $10 \%$ et $4 \%$ ont été récemment développées par SongréOuattara et al. (2016) au Burkina Faso pour servir d'aliment véhicule. Cependant, très peu d'informations existent sur leur durée de vie et leurs conditions de conservation. Ainsi, notre objectif était d'évaluer l'effet de deux types d'emballage sur la qualité nutritionnelle et la stabilité des biscuits de sorgho enrichis au moringa et à la spiruline au cours du temps à travers les paramètres de qualité en comparaison avec un biscuit témoin non enrichi.

de spiruline achetée auprès de la ferme de production de Loumbila, ville située à $20 \mathrm{~km}$ de la capitale. La poudre de gomme arabique provenait de l'atelier de production du DTA.

Production de biscuits de sorgho enrichis et non enrichis: Trois types de biscuits de sorgho étaient produits selon le procédé de Songré-Ouattara et al. (2016). Un type non enrichi considéré comme biscuit témoin et deux types enrichis par addition de $10 \%$ de poudre de feuilles séchées de moringa et de $4 \%$ de poudre de spiruline utilisant différents ingrédients, notamment la matière grasse végétale, le sucre, les œufs, la poudre de gomme arabique, le lait en poudre, la poudre levant, le sucre vanillé et le sel comme montré dans le Tableau 1. Toutes ces productions étaient réalisées en triple. 
Tableau 1 : Formulation des biscuits de sorgho

\begin{tabular}{lccc}
\hline Ingrédients (\%) & $\begin{array}{c}\text { Biscuits de sorgho non } \\
\text { enrichis }\end{array}$ & $\begin{array}{c}\text { Biscuits de sorgho } \\
\text { enrichis au moringa }\end{array}$ & $\begin{array}{c}\text { Biscuits de sorgho } \\
\text { enrichis à la spiruline }\end{array}$ \\
\hline Farine de sorgho & 52,9 & 42,9 & 48,9 \\
Poudre de moringa & 0 & 10 & 0 \\
Poudre de spiruline & 0 & 0 & 4 \\
Matière grasse végétale & 20 & 20 & 20 \\
Sucre & 11 & 11 & 11 \\
OEufs & 7,3 & 7,3 & 7,3 \\
Poudre de gomme arabique & 4 & 4 & 4 \\
Lait en poudre & 1,7 & 1,7 & 1,7 \\
Poudre levante & 1,7 & 1,7 & 1,7 \\
Sucre vanillé & 1,3 & 1,3 & 1,3 \\
Sel & 0,1 & 0,1 & 0,1 \\
\hline
\end{tabular}

Conservation et stockage des biscuits : Chacun des trois types de biscuits produits ont été refroidis et ensuite emballés dans deux types d'emballage, notamment le plastique et l'aluminium. Ils ont été scellés hermétiquement à l'aide d'une thermo-soudeuses et ensuite conservés dans une salle à température ambiante $\left(28-30^{\circ} \mathrm{C}\right)$ à l'abri de la lumière.

\section{Analyses physico-chimiques et nutritionnelles}

Les analyses de suivi de conservation ont été réalisées mensuellement pendant les premiers mois, puis ensuite

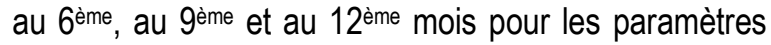
physico-chimiques et nutritionnels ci-après cités.

Teneurs en eau : La teneur en eau des échantillons a été déterminée par pesée différentielle avant et après passage à l'étuve à $130^{\circ} \mathrm{C}$ pendant $2 \mathrm{~h}$ selon la norme française (NF V 03-707, 2000).

Teneurs en lipides: Les teneurs en lipides des échantillons ont été déterminées par la méthode d'extraction au Soxhlet selon la norme internationale (ISO 659 : 1998), avec l'hexane comme solvant.

Teneurs en protéines totales : Les teneurs en protéines totales ont été déterminées par la méthode de Kjeldahl selon la norme française (NF V03-050 : 1970).

Teneurs en cendres totales : Les teneurs en cendres ont été déterminées par incinération dans un four à moufle (Nabertherm ${ }^{\circledR}$ ) à $550^{\circ} \mathrm{C}$ pendant $4 \mathrm{~h}$ selon la norme internationale (ISO 2171 : 2007).

Teneurs en glucides totaux : Les teneurs en glucides totaux ont été déterminées par différence (Egan et al. 1981) selon la formule : Teneur en glucides totaux $(\%)=$ 100 - [teneur en eau $(\%)+$ teneur en protéine $(\%)+$ teneur en lipide (\%) + teneur en cendre (\%)].
Valeurs énergétiques : Les valeurs énergétiques des aliments de complément ont été calculées en utilisant les coefficients d'Atwater et Benedict (1899) selon la formule suivante :

Valeur énergétique $(\mathrm{Kcal} / 100 \mathrm{~g})=[$ teneur en glucides $(\%)$ $\times 4(\mathrm{Kcal})+$ teneur en protéines $(\%) \times 4(\mathrm{Kcal})+$ teneur en lipides $(\%) \times 9($ Kcal $)]$.

Détermination du taux d'acidité grasse: L'acidité grasse des échantillons de biscuits a été déterminée par extraction des acides gras libres utilisant l'éthanol à $95 \%$ à la température ambiante du laboratoire selon la norme française (NF ISO 7305 :1998) suivie d'une titration par une solution d'hydroxyde de sodium $\mathrm{N} / 9$. Les résultats ont été exprimés en $\mathrm{g}$ d' $\mathrm{H}_{2} \mathrm{SO}_{4}$ pour $100 \mathrm{~g}$ de produit.

Détermination de l'indice de peroxyde: L'indice de peroxyde a été déterminé selon la méthode Association of Official Analytical Chemists (AOCS Cd 8-53:1998). Les lipides des biscuits stockés étaient extraient utilisant I'hexane et titrés par une solution de thiosulfate $(0,1 \mathrm{~N})$ en présence d'une solution éthanolique d'iodure de potassium. Les résultats sont exprimés en $\mathrm{meqO}_{2} / \mathrm{kg}$ pour $100 \mathrm{~g}$ de biscuit. Les résultats sont exprimés en $\%(\mathrm{~m} / \mathrm{m})$ d'équivalent acide oléique.

Analyses microbiologiques : Pour toutes les analyses microbiennes, $10 \mathrm{~g}$ de d'échantillon de chaque type de biscuit ont été prélevés à intervalle de 30 jours pendant les trois premiers mois, puis tous les trois mois pendant une durée totale de 12 mois et ont été homogénéisés dans $90 \mathrm{ml}$ d'eau peptonée stérile utilisant un stomacher 400 (Type BA 7021, England). La flore totale ou mésophile a été déterminée selon la norme française (NF EN ISO 4833 : 2003) utilisant une étuve à $30^{\circ} \mathrm{C}$ pendant $72 \mathrm{~h} \pm 3 \mathrm{~h}$. Les coliformes totaux ont été déterminés selon 

qualité nutritionnelle et microbiologique des biscuits de sorgho enrichis au moringa et à la spiruline

la Norme Internationale (ISO 4832 : 2006) utilisant une étuve à $37^{\circ} \mathrm{C}$ pendant $24 \mathrm{~h} \pm 2 \mathrm{~h}$. Les coliformes thermotolérants ont été déterminés selon la norme française (NFV08-060 : 2010) utilisant une étuve à $44^{\circ} \mathrm{C}$ pendant $24 \mathrm{~h} \pm 2 \mathrm{~h}$. Les levures et moisissures ont été déterminés selon la norme française (NF ISO 7954, 1988) utilisant une étuve à $25^{\circ} \mathrm{C}$ pendant 5 jours. Les colonies ont été comptées après la période d'incubation et les résultats ont été exprimés en log cfu/g.

\section{RESULTATS ET DISCUSSION}

Évolution de la composition globale des biscuits de sorgho enrichis au cours du temps: La Figure 1 présente l'évolution au cours du temps des paramètres physico-chimiques et nutritionnels des différentes formulations de biscuits enrichis conditionnés dans deux types d'emballages, l'aluminium et le plastique en comparaison au biscuit témoin. Le taux d'humidité initial varie significativement $(p<0,05 \%)$ entre les différents types de biscuits, de $2,7 \%$ à $5,5 \%$ avec les plus grandes valeurs pour les biscuits enrichis au moringa, soit supérieures à $5 \%$, limite maximale suggérée par Zydembos et Humphrey-Taylor (2003) pour assurer une longue conservation. Cette différence pourrait se révéler être la conséquence du taux d'incorporation élevé (10\%) et ou du taux d'humidité élevé de la poudre de moringa, soit de 9,28 $\pm 0,71 \%$ (Kaboré, 2012) ou encore des conditions de cuisson. L'évolution des taux d'humidité des trois types de biscuits durant les douze mois de conservation montre qu'il y'a une très faible augmentation non significative $(p>0,05 \%)$ pour un même type de biscuit et pour chaque condition d'emballage étudié, excepté pour les biscuits au moringa. En effet, avec les emballages de type aluminium et de type plastique, les taux d'humidité observés en fin de conservation sont respectivement de $3,15 \%$ et $3,33 \%$ pour les biscuits témoins, de $3,65 \%$ et $3,60 \%$ pour les biscuits à la spiruline en accord avec ceux rapportés par Tasnim Farzana et Suman Mohajan (2015) et Yadav et al. (2015) alors qu'ils sont varient entre $5,21 \%$ et $5,38 \%$ pour les biscuits au moringa. Ces faibles variations enregistrées entre les taux d'humidité initiaux et finaux des biscuits dépendent du taux de transmission de la vapeur d'eau de l'emballage et des conditions de stockage. Cependant, même si ces faibles taux d'humidité des biscuits présagent une bonne conservation, il n'en est pas moins que ces derniers sont capable de développer une rancidité oxydative et une rancidité hydrolytique (Prasanth Kumar et al., 2012). En outre, une comparaison des deux types d'emballage étudiés, notamment le plastique et l'aluminium laisse penser qu'lls ont des capacités
Analyses statistiques : Les paramètres à l'étude ont été examinés à l'aide de statistiques descriptives (moyennes, écarts types). Les données ont été soumises à une analyse de variance (ANOVA) à l'aide du logiciel SPSS ${ }^{\mathrm{TM}}$ Statistics version 20.0 (IBM, USA) et la comparaison des moyennes a été réalisée selon le test de Newman-Keuls au seuil de $5 \%$.

comparables quant à leur imperméabilité à l'eau (capacité à réduire la perméabilité à l'eau et à l'oxygène, composés volatiles ou gazeuses ; rôle de barrière satisfaisant). Les teneurs en protéines sont significativement $(p<0,05 \%)$ plus élevées pour les biscuits de sorgho enrichis, variant au cours du temps respectivement entre $5,7 \%$ et $6,8 \%$ avec la spiruline et entre $5,6 \%$ et $6,8 \%$ avec le moringa, contre une variation comprise entre $4,2 \%$ et $5 \%$ pour le biscuit témoin, indépendamment du type d'emballage. Ces résultats mettent en évidence de très faibles différences entre les teneurs en protéines pour tous les deux types d'emballages durant la période de conservation. Des valeurs similaires de teneurs en protéines des biscuits sont rapportées par Okpala et al. (2013). Cependant, ces teneurs en protéines sont en dessous de celles antérieurement rapporté par SongréOuattara et al. (2016) ce qui pourrait être expliqué par une variation au niveau de la production de matière sèche des espèces de moringa et de spiruline ou des conditions opératoires d'analyses. De même, ces valeurs de protéines sont très faibles que celles rapportées par Agu et Okoli (2014) et Tasnim Farzana et Suman Mohajan (2015) pour des biscuits de céréales enrichis. Pour les lipides, tous les différents types de biscuits de sorgho stockés ont des teneurs qui varient entre $19,6 \%$ et $21,1 \%$ et les teneurs les plus élevées sont observées avec les biscuits de sorgho enrichis à la spiruline (19,8\% et $20,5 \%)$ et en particulier au moringa (20,4\% et $21,1 \%)$. Par ailleurs, pour toutes les conditions de conservation, l'évolution des teneurs en lipides des différents types de biscuits ne change pas significativement au cours du temps et montre la même allure. Les teneurs en cendres pour tous les échantillons de biscuits de sorgho stockés varient entre $2,4 \%$ et $3,6 \%$ avec des teneurs significativement $(p<0,05 \%)$ plus élevées pour les biscuits de sorgho enrichis à la spiruline $(2,9 \%$ et $3,1 \%)$ et au moringa $(3,4 \%$ et $3,6 \%)$ qui sont en effet de bonne source de minéraux (Songré-Ouattara et al., 2016). Toutefois, on note une faible décroissance des teneurs en minéraux pendant la période de conservation, notamment avec le 
Songré-Ouattara et al ., J. Appl. Biosci. 2017 Impact de l'emballage et de la durée de conservation sur la qualité nutritionnelle et microbiologique des biscuits de sorgho enrichis au moringa et à la spiruline

biscuit témoin et les biscuits enrichis au moringa, passant respectivement de $2,7 \% \%$ à $2,4 \%$, et de 3,6 à $3,4 \%$. Ainsi, seules les teneurs en matières minérales des biscuits à la spiruline restent quasiment constantes durant la conservation, soit environ 3\%. Concernant les glucides, les teneurs sont plus élevées dans le biscuit témoin variant entre $72,6 \%$ et $73,1 \%$ et plus faibles dans les biscuits enrichis du fait de la supplémentation avec la spiruline et le moringa, avec respectivement des teneurs comprises entre $69,9 \%$ et $71,2 \%$, puis entre $68,4 \%$ et $70,4 \%$. L'évolution durant les douze mois de conservation montre une quasi-stabilité de la teneur en glucides pour chaque type de biscuit pour toutes les conditions de conservation.
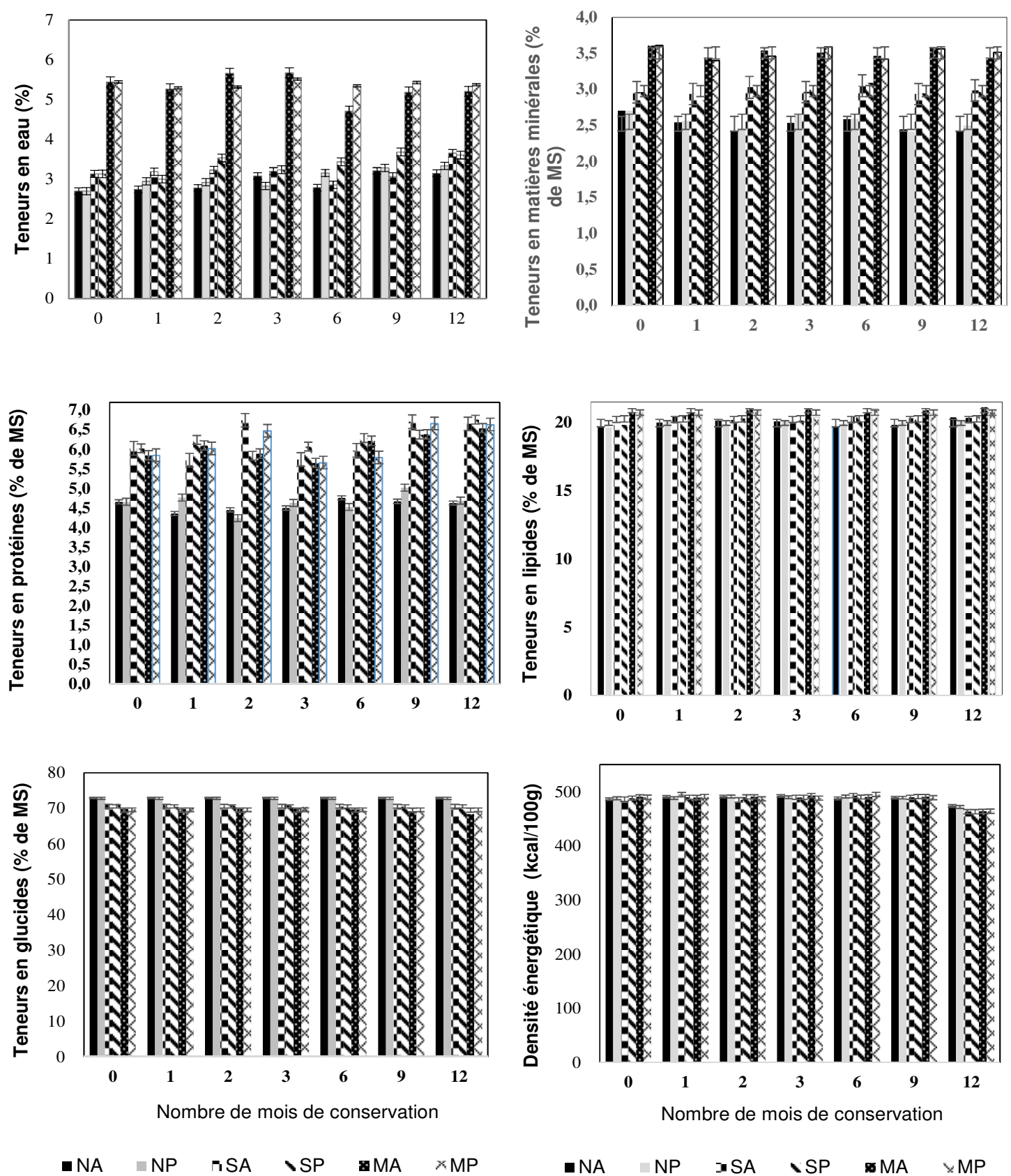

Figure 1 : Évolution de la composition globale des biscuits de sorgho enrichis au cours du temps 
Évolution de l'acidité grasse et l'indice de peroxyde : La Figure 2 montre l'évolution du taux d'acidité grasse et de l'indice de peroxyde dans les biscuits de sorgho au cours du stockage. Ces composés sont formés dus à l'oxydation des triglycérides (Nagi et al., 2012). L'évolution du taux d'acidité ne montre pas de variation significative pour chaque type de biscuit et selon l'emballage étudié au cours du stockage des biscuits. Ces taux d'acidité grasse sont faibles ce qui indique une faible oxydation des matières grasses comparés aux taux d'acidité plus élevés rapportés par Prasanth Kumar et al. (2012) lors de la conservation des biscuits, soit variant entre $0,36 \%$ et $0,60 \%$. Toutefois, les taux d'acidité grasse sont significativement $(p<0,05)$ plus élevés dans les biscuits de sorgho au moringa, soit compris entre 0,1 et $0,12 \mathrm{~g}$ de $\mathrm{H}_{2} \mathrm{SO} 4$ pour $100 \mathrm{~g}$ de biscuit tandis que dans les biscuits à la spiruline et le biscuit témoin, ils sont très bas, respectivement compris entre 0,03 et $0,05 \mathrm{~g}$ de $\mathrm{H}_{2} \mathrm{SO} 4$ pour $100 \mathrm{~g}$ de biscuit et entre 0,02 et $0,03 \mathrm{~g}$ de $\mathrm{H}_{2} \mathrm{SO} 4$ pour $100 \mathrm{~g}$ de biscuit. L'indice de peroxyde qui renseigne également sur l'altération de la matière grasse est de valeur plus élevée à celles rapportés par Prasanth Kumar et al. (2012) et notamment pour les biscuits les plus riches en matières grasses, particulièrement ceux enrichis au moringa comparativement au biscuit témoin. Ces valeurs d'indice de peroxyde sont très variables durant la conservation, soit entre 3 et $17 \mathrm{meqO}_{2} / \mathrm{kg}$ d'huile.

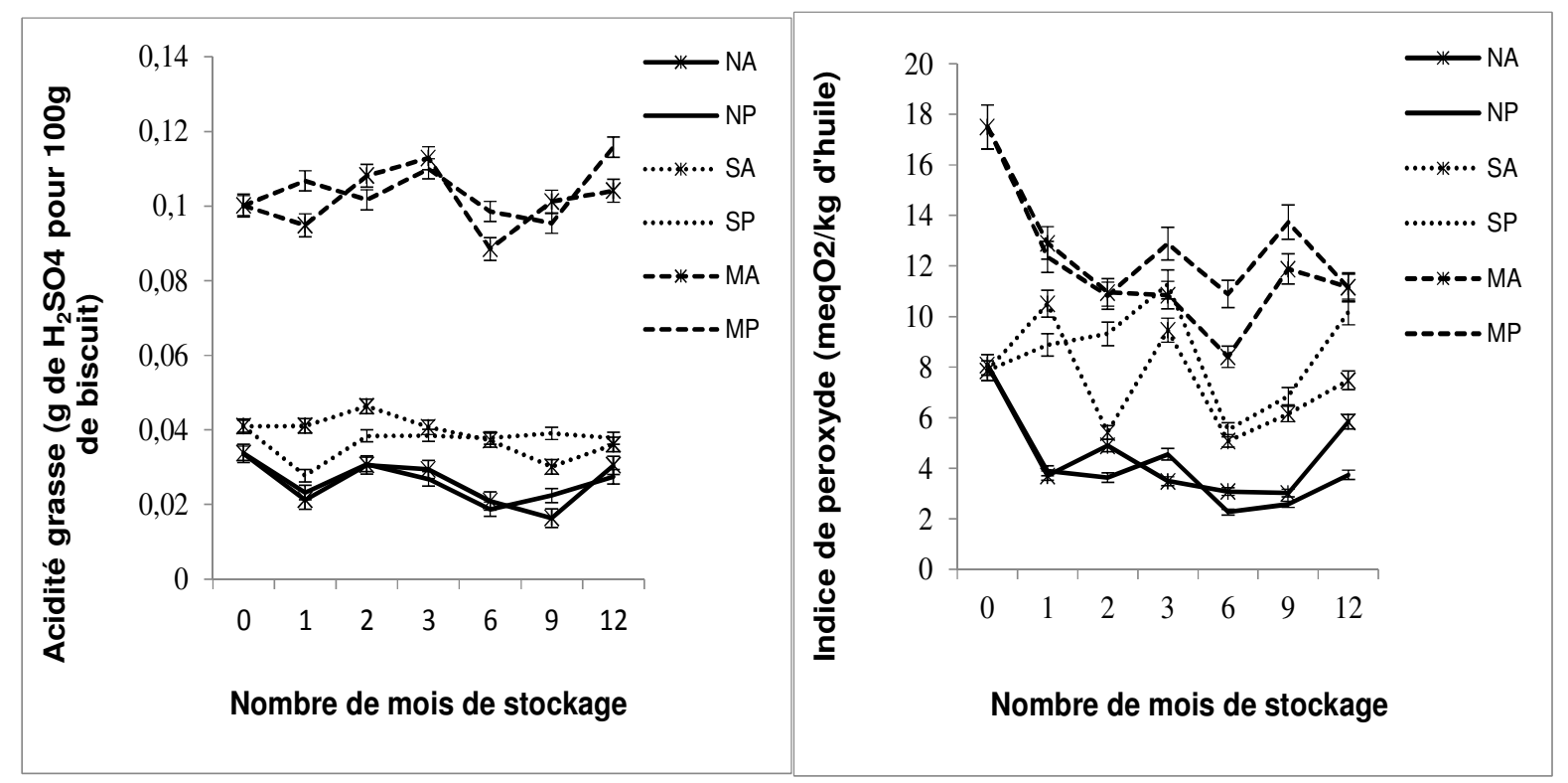

Figure 2 : Évolution au cours du temps de l'acidité grasse et de l'indice de peroxyde dans les différents types de biscuits

Évolution de la flore microbienne dans les biscuits durant la conservation : La Figure 3 montre l'évolution de la flore mésophile des trois types de biscuit durant la conservation. Elles sont à des concentrations différentes et très variables pendant les premiers mois au douzième mois de conservation et se situent entre 1,9 et 5,8 log (cfu/g) et les concentrations les plus élevées sont observées dans les biscuits enrichis au moringa (2,9 et $5,8 \log (\mathrm{cfu} / \mathrm{g}))$ et à la spiruline (2,3 à 5,3 log (cfu/g)) tandis que dans le biscuit témoin, les plus fortes concentrations sont à $3,8 \log (\mathrm{cfu} / \mathrm{g})$. La présence de la flore mésophile est probablement due à une contamination post transformation, notamment des emballages utilisés et ou du non-respect des bonnes pratiques d"hygiène de conditionnement. Agu et Okoli (2014) ont rapporté après 20 jours de conservation de biscuit, une flore bactérienne de 3,6 log (cfu/g) et de moisissures de 4,69 log (cfu/g). Toutefois, on note que la concentration de la flore aérobie mésophile des biscuits est à la limite de la norme concernant les critères microbiologiques applicables aux denrées alimentaires (CE, 2005), soit entre 5 et $6 \log$ (cfu/g). Pendant les 12 mois de conservation, les coliformes totaux et thermotolérants sont de concentrations très faibles, soit moins de $1 \log (\mathrm{cfu} / \mathrm{g}$ ) dans tous les différents types de biscuits et selon l'emballage utilisé. Ce résultat peut être 
Songré-Ouattara et al ., J. Appl. Biosci. 2017 Impact de l'emballage et de la durée de conservation sur la qualité nutritionnelle et microbiologique des biscuits de sorgho enrichis au moringa et à la spiruline

expliqué par une bonne pratique d'hygiène durant la production des biscuits et par la destruction de la flore microbienne durant la cuisson $\left(135^{\circ} \mathrm{C}\right.$ pendant $\left.40 \mathrm{~min}\right)$. La charge en levures et en moisissures est restée constante durant les 12 mois de conservation dans tous les types de biscuits et toutes les conditions de conservation étudiées, soit moins de $1 \mathrm{log}(\mathrm{cfu} / \mathrm{g})$ en accord avec les travaux de Kaboré (2012) sur les biscuits de patate douce à chair orange (PDCO) enrichis à la spiruline.

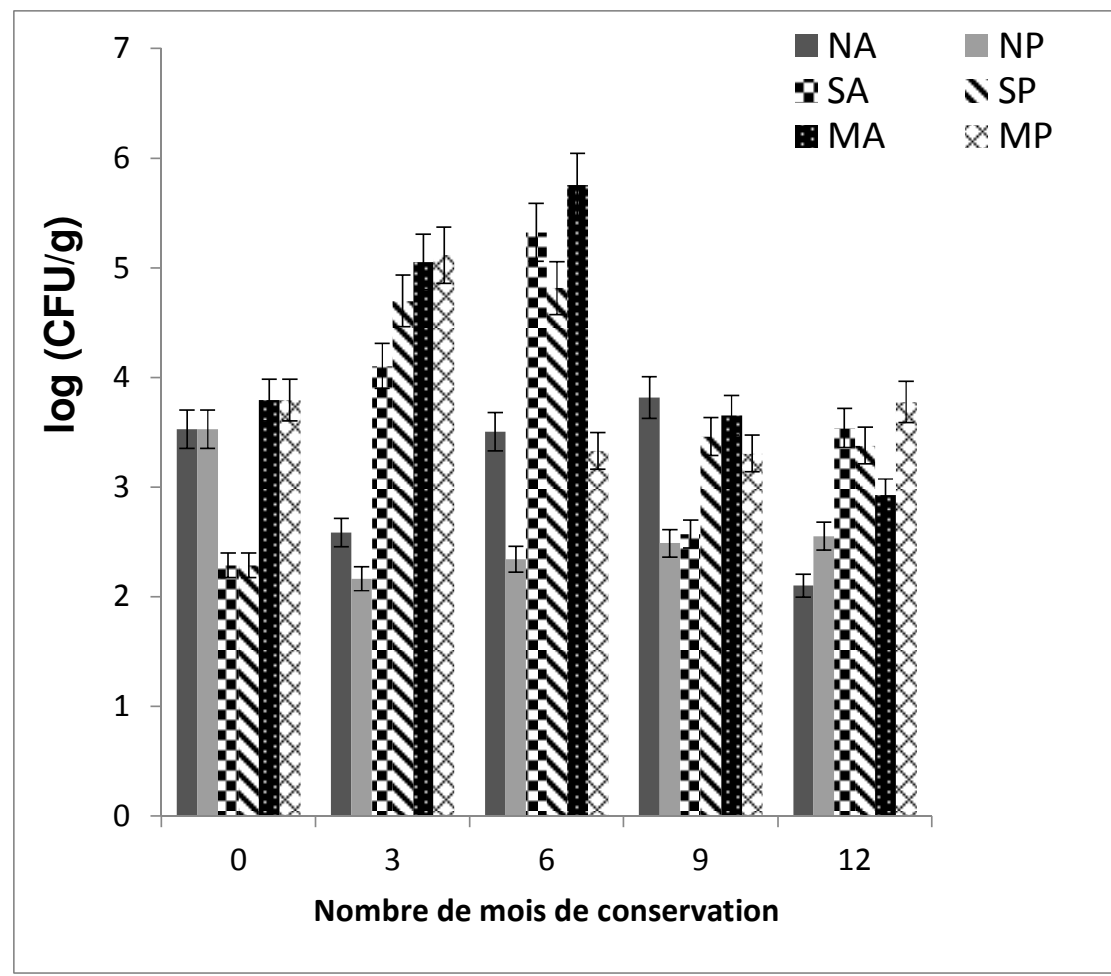

Figure 3 : Évolution de la flore mésophile dans les différents types de biscuits durant la conservation

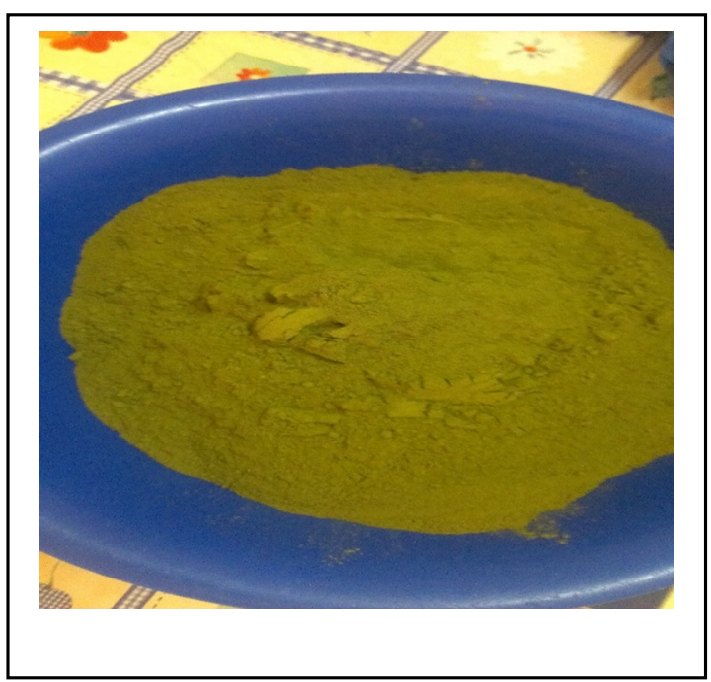

Photo 1 : Poudre de moringa

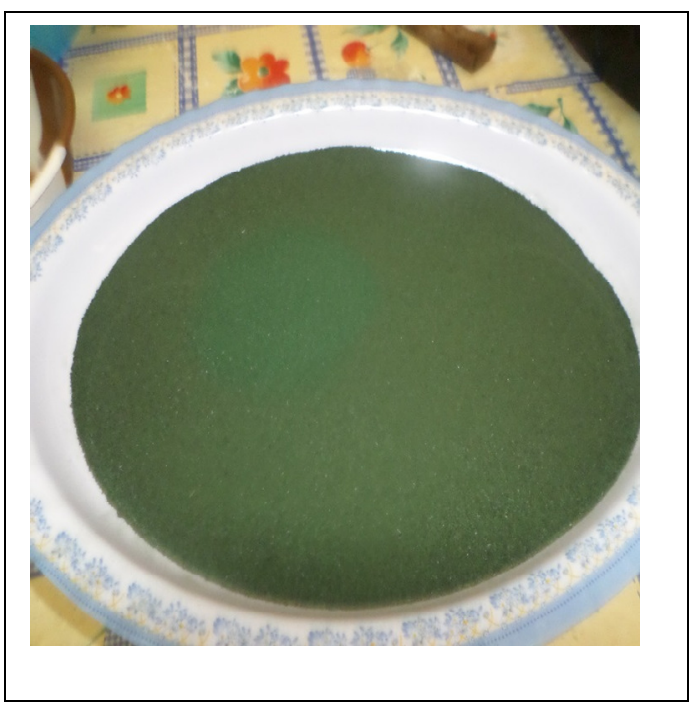

Photo 2 : Poudre de spiruline

\section{CONCLUSION}


Les paramètres nutritionnels des biscuits étudiés selon l'emballage sont restés stable pendant 12 mois de conservation. En effet, II n'a pas été observé une influence de l'emballage sur les concentrations des différents nutriments analysés comme l'humidité, les protéines, les lipides, les cendres et les glucides. Sur le plan de qualité microbiologique des différents types de biscuits, les levures et moisissures ainsi que les coliformes totaux et fécaux sont restés stables et de concentrations très faibles. En revanche une assez importante concentration de la flore mésophile a été mise en évidence et dont l'évolution n'est pas attribuable aux conditions de conservation mais plutôt à des contaminations post production des biscuits.

\section{CONFLIT D'INTERET}

Les auteurs déclarent l'absence de conflit d'intérêt.

\section{REMERCIEMENTS}

Ces travaux ont été financés par le Fonds National de la Recherche et de l'Innovation pour le Développement à travers le projet d'Appui à la production de Biscuits et par la Fondation Internationale pour la Science (Grant N $\left.{ }^{\circ} E / 3944-2 F\right)$.

\section{REFERENCES}

AFNOR (Association Française de Normalisation), 1970. Directives générales pour le dosage de l'azote avec minéralisation selon la méthode de Kjedahl. Produits Agricoles Alimentaires, NF V 03-050.

AFNOR (Association Française de Normalisation), 2000. Détermination de la teneur en eau, méthode pratique. Céréales, Légumineuses, Produits Dérivés, NF V 03-707.

AFNOR (Association Française de Normalisation), 2010. Microbiologie des aliments. Dénombrement des coliformes thermotolérants, techniques par comptage des colonies à $44^{\circ} \mathrm{C}$, NF V08-060.

Agu, HO, Ayo JA, Paul AM. Folorunsho F, 2007. Quality characteristics of biscuits made from wheat and African breadfruit (Treculia Africana). Nigerian Food Journal 25:19 - 25.

Agu HO, Okoli NA, 2014. Physico-chemical, sensory, and microbiological assessments of wheat-based biscuit improved with beniseed and unripe plantain Food Science \& Nutrition 2(5):464-469. doi: $10.1002 / f s n 3.135$.

AOCS (1998) Official methods of the American Oil Chemists Society, 4th edn. AOCS method no. Ac 2-41, (1997) The AOCS Official Butt-tube Method Ac 3-44, AOCS method Nos. Ca 5a-40 (1998) AOCS method Nos. Cd 8-53 (1998) American Oil Chemists Society, Champaign, IL, USA

Atwater WO, Benedict FG. 1899. Experiments on the metabolism of matter and energy in the human body.US Department of Agriculture. Washington, D.C. Bulletin 69, 112p.

Chakraborty SK, Kumbhar BK, Chakraborty S, Yadav P, 2011. Influence of processing parameters on textural characteristics and overall acceptability of millet enriched biscuits using response surface methodology. Journal of Food Science and Technology 48(2):167-174.

Egan H, Kirk RS, Sawyer R, 1981. Pearson's Chemical Analyses of Food (8th edition). Churchill. Livingstone : London-UK;591p.

Gajera HP, Kapopara MB, Patel VH, 2010. Application of peanut butter to improve fatty acid composition of biscuits. Journal of Food Science and Technology 47(3):285-289

Handa C, Goomer S, Siddhu A, 2012. Physicochemical properties and sensory evaluation of fructoligosaccharide enriched cookies. Journal of Food Science and Technology 49(2):192-199

INSD (Institut National de la Statistique et de la Démographie). 2012. Enquête Démographique et de Santé et à Indicateurs Multiples du Burkina Faso 2010. Calverton, Maryland, USA : INSD. $501 p$.

ISO (International Standardization Organization), 1998. Détermination de la teneur en matière grasse selon la méthode d'extraction par Soxhlet. ISO 659.

ISO (International Standardization Organization), 2003. Directives générales pour le dénombrement des levures et moisissures, techniques par comptage des colonies à $25^{\circ} \mathrm{C}$, ISO 7954 .

ISO (International Standardization Organization), 2003. Microbiologie des aliments. Méthode horizontale pour le dénombrement des micro-organismes; technique de comptage des colonies à $30^{\circ} \mathrm{C}$, ISO 4833.

ISO (International Standardization Organization), 2006. Microbiologie des aliments-Méthode horizontale 
pour le dénombrement des coliformes- Méthode par comptage des colonies, ISO 4832.

ISO (International Standardization Organization), 2007. Dosage du taux de cendre par incinération à $550^{\circ} \mathrm{C}$. Céréales, légumineuses et produits dérivés. ISO 2171.

Kaboré N, 2012. Optimisation de la production de biscuits à base de patate douce à chair orange. Mémoire de licence.76p.

Manley D, 2000. Technology of biscuits, crackers and cookies, $3^{\text {rd }}$ edn. CRC Press Woodhead Publishing Ltd, Cambridge.

Nagi HPS, Kaul J, Dar BN, Sharma S, 2012. Effect of storage period and packaging material on shelf life of cereal bran incorporated biscuits. American Journal of Food Technology 7(5): 301310.

O'Brien CN, Champmemb O, Nexille DP, Kengh MK, Arendt EK, 2003. Effect of varying microencapsulation process on the functionality hydrogenated vegetable fat in short dough biscuit. Food Research International, 36:215221.

Okpala L, Okoli E, Udensi E, 2013. Physico-chemical and sensory properties of cookies made from blends of germinated pigeon pea, fermented sorghum, and cocoyam flours. Food Science \& Nutrition 1(1): 8-14, doi10.1002/fsn3.2.
Prasanth Kumar PK, Sai Manohar R, Indiramma AR, Gopala Krishna AG, 2014. Stability of oryzanol fortified biscuits on storage. Journal Food Science and Technology 51(10):2552-2559, doi 10.1007/s13197-012-0712-1.

Songré-Ouattara LT, Gorga K, Bationo F, Savadogo A, Diawara B, 2016. Utilisation du moringa, de la spiruline, de la patate douce à chair orange et d'un complexe minéral et vitaminique dans la fabrication de biscuits de sorgho enrichis destinés aux jeunes enfants. International Journal of Biological and Chemical Sciences, 10(4) 1651-1665, doi: http://dx.doi.org/10.4314/ijbcs.v10i4.17

Tasnim Farzana, Suman Mohajan. 2015. Effect of incorporation of soy flour to wheat flour on nutritional and sensory quality of biscuits fortified with mushroom. Food Science \& Nutrition 3(5): 363-369, doi 10.1002/fsn3.228=

Yadav RB, Yadav BS, Dhull N. 2012. Effect of incorporation of plantain and chickpea flours on the quality characteristics of biscuits. Journal Food Science and Technology 49(2):207-213, doi 10.1007/s13197-011-0271-x.

Zydenbos S, Humphrey-Taylor V, 2003. Biscuits, cookies, and crackers New Zealand Institute for Crop \& Food Research Limited, Christchurch, New Zealand Copyright 2003, Elsevier Science Ltd. All Rights Reserved. 